\title{
Objective Assessment Technologies: General Guidelines for Scar Assessment
}

Julian Poetschke and Gerd G. Gauglitz

\section{Contents}

16.1 Background - 144

16.2 Choosing the Right Tools for Each Scar - 144

16.3 Optimizing the Measurement Process - 144

16.3.1 Preparing the Surroundings - 144

16.3.2 Configuring and Calibrating the Assessment Tools - 145

16.3.3 Preparing the Patient -145

16.3.4 Performing the Measurements -145

16.4 Interpreting Therapeutic Success with Objective Scar Assessment Technologies - 146

16.4.1 Data Assessment and Evaluation - 146

16.4.2 Clinically Important Difference - 146

16.5 Conclusion - 146

References - 147 


\subsection{Background}

Comprehensive scar treatment is becoming more and more readily available. Both the awareness of medical professionals regarding the impairments of severely scarred patients and their knowledge of treatment options for scars have increased significantly during recent years. Those developments have been made possible by extensive research of new treatment options and continued reevaluation of long-established ones.

However, large numbers of studies continue to rely predominantly on subjective evaluation methods like scales and questionnaires to gauge the success of their treatment. Objective scar evaluation technology, too, has shown an immense development throughout recent years, and researchers and clinicians alike can now choose from a large arsenal of options to document treatment progress and failure [1].

But while this newfound selection of tools for objective scar analysis has helped to improve research, it has also introduced new problems.

With many different manufacturers offering many different devices for analysis of a vast number of parameters, researchers are left with the dilemma to choose the right tools for their individual projects with little evidence-based recommendations available. Oftentimes, devices will not be able to measure in standardized units but will provide measurement results in the manufacturer's own proprietary format that offers little comparability with results from different research groups with different measurement tools. The process of measuring the desired parameters reliably in the oftentimes hectic environment of everyday clinical routine can be daunting too and can lead to problems with recreating measurements and making sure that repeated measurements yield comprehensible results.

In this chapter, we want to focus on the basics of objective measurement technologies that allow researchers and clinicians to find the right tools for their desired analyses, to make comprehensive plans about how to document their patients' scars in a way that can both easily and reliably be reproduced, and to understand the value and impact of the results of their measurements.

Obtaining and cultivating this knowledge can then not only lead to improving the level of evidence in scar research but also help in everyday clinical treatment scenarios where in-depth scar analysis might provide physicians with a sensitive gauge for the success or failure of their applied treatment.

\subsection{Choosing the Right Tools for Each Scar}

Before planning a research project or the documentation of clinical results in scar therapy based on objective assessment technology, care must be taken to identify the tools best suited for each specific undertaking. Since scar documentation as well as the consecutive sorting, assessment, and evaluation of data is considerably time-consuming, superfluous measurements should be avoided.

The first step is identifying the scar parameters that require documentation and afterward choosing the tools suitable to assess the development of the chosen parameters. When trying to assess the influence of a certain treatment option on scars, parameters most likely affected positively or negatively (e.g., side effects) should be selected.

When choosing the most suitable assessment technology, a thorough literature research should be performed to identify assessment options that have been well established and are used by other researchers as well to ensure both the suitability for the task and, later, comparability of the results between different research groups.

Ideally, comparability does not require different researchers to use identical hardware but rather that different hardware uses the same units of measurement. While this is common in ultrasound technology where most, if not all, units are able to perform measurements based on SI units, more specialized tools for profilometry or colorimetry specifically designed for application in aesthetic medicine often employ proprietary units of measurement that are difficult to compare with other researches.

While this does not necessarily mean those options are unsuited for research applications, it should be taken into consideration beforehand.

\subsection{Optimizing the Measurement Process}

\subsubsection{Preparing the Surroundings}

The room where measurements are conducted should be light, cool, and dry. Illumination through natural light should be minimized so that it does not influence photographic and colorimetric testing where changing outside lighting could act as a disruptive factor. The ambient temperature should neither be too warm or too cool since physiological reactions to extreme temperature (e.g., sweating, goose bumps) can influence measurements, especially regarding physiological skin parameters. Furthermore, extreme temperatures, just like humidity, could also influence the functionality and longevity of the measurement instruments. Some manufacturers even provide ambient condition sensors to monitor parameters like temperature or humidity and save the values together with the measured results. This can guarantee both superior awareness of the lab ambience as well as improve comparability of the achieved measurement results. 


\subsubsection{Configuring and Calibrating the Assessment Tools}

Reliable measurements are paramount in every clinical and/or research setting. This starts with making sure that the assessment instruments are properly cared for and calibrated.

Most devices come with extensive instructions on how to calibrate them, and adhering to those recommendations is important since even small discrepancies in the measurement process can yield significantly altered results, especially with modern, highly sensitive equipment.

Instruments that have direct contact with the skin, for example, devices that measure skin elasticity, color, or physiological properties, should be cleaned regularly as sebum, hairs, and makeup can accumulate on the measuring probes and alter measurements as well as hinder successful calibration.

Photography-based systems require regular cleaning too, so that there are no specks on the lens or projector systems (e.g., PRIMOS system).

Most devices allow for a variety of parameters to be configured that can greatly influence how the measurements are conducted and what the results are going to be. Those parameters need to be defined beforehand and require standardization so that subsequent measurements can be compared. Photography-based devices that use different exposure times or white balances during measurements will result in significantly different looking images, skin elasticity values will differ greatly when on and off times of the suction probes of the Cutometer are changed, and the same is true for many other devices, too. Especially when more than one researcher is working with a certain device, care should be taken to check all the relevant parameters before measurements are performed.

\subsubsection{Preparing the Patient}

The areas to be measured should be clean and free of makeup, skin-care products, or therapeutic agents (like ointments, gels, silicon sheets).

Throughout the measurement process, patients should remain in a neutral, predefined position so as not to alter certain measurement results due to their pose. Especially during measurements regarding skin texture and relief or elasticity, identical positioning for subsequent measurements is crucial in ensuring comparability of the results. Scars that run close to or over joints are greatly affected when those joints are moved, and elasticity results, for example, might reveal drastically different results when said joints are fully flexed or extended. It can therefore help when the positioning of the patient is predefined (e.g., patient standing, arms hanging loosely on his sides) and said positioning is kept throughout all measurements (• Fig. 16.1).

\subsubsection{Performing the Measurements}

After all the preparations have been made, calibrations have been performed, and configurations have been checked, the measurements are performed. Here, it is important to ensure subsequent measurements are performed similarly. Camera-based systems often provide integrated overlay functions that allow the examiner to achieve identical captures during successive assessments, and some devices offer integrated software solutions to stack subsequently taken images to only measure the common areas; however sometimes, such solutions are not offered. It can therefore help to define anatomical landmarks for orientation and to fashion templates so that positioning of the measuring probe or the camera system remains constant [2].
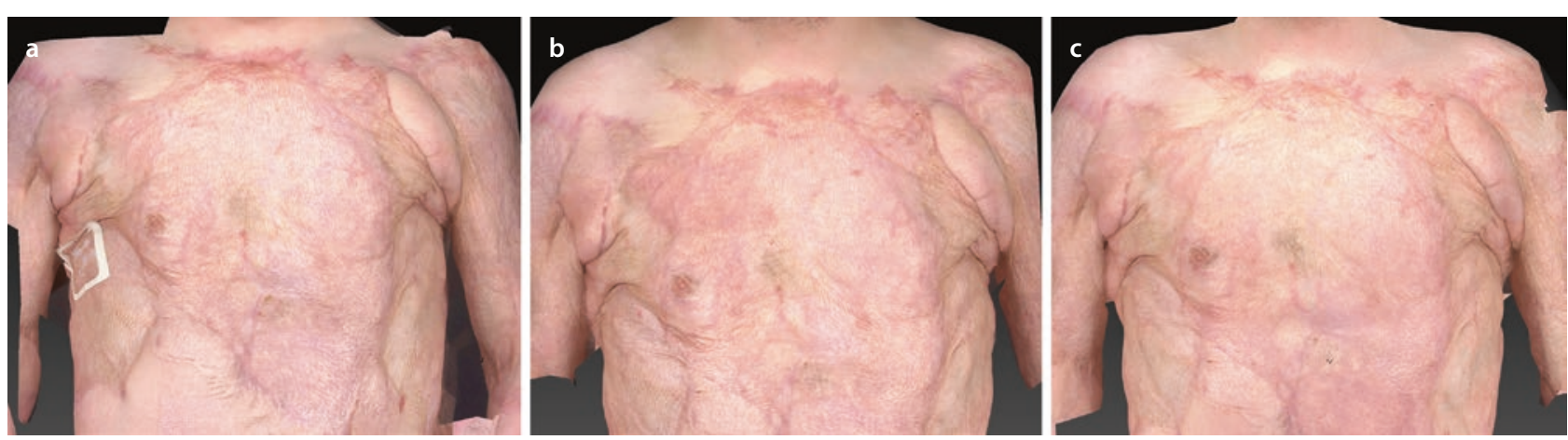

- Fig. 16.1 Canfield Vectra X3 imaging before a, 3 months after b, and 6 months after $\mathbf{c}$ one session of fractional ablative laser treatment of the upper right chest. The patient had been instructed to maintain a standardized pose (standing upright, arms hanging loosely by his sides, facing forward) to ensure comparability between subsequent images 


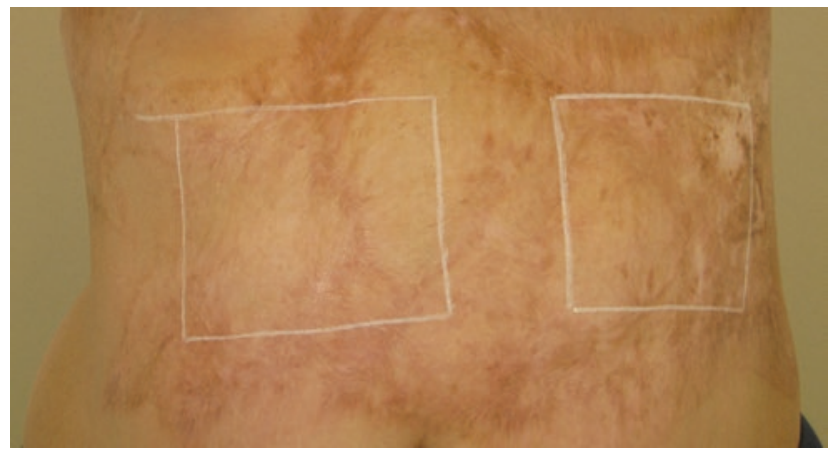

- Fig. 16.2 Severe burn scars on the abdomen of a female patient. Note the marked squares where one has been defined as the treatment and the other as the control area. By using a foil template and anatomical landmarks, those areas can be retraced for followup measurements which are always conducted within the marked squares to avoid confounding of the results. This course of action ensures that surface profilometry and skin elasticity measurements are always performed on the same area

Devices that measure skin elasticity or color often rely on small probes with relatively small openings through which the individual parameters are measured. This often proves challenging for the examiner since exact repositioning for a subsequent measurement often is difficult if not impossible. Furthermore, especially in widespread scarring, getting representative results from only one measurement is virtually impossible, especially if one cannot ensure that measurements are always taken at the same spot.

These problems, unfortunately, cannot be addressed satisfactorily with devices that are not able to measure the entire scarred surface, and therefore, a compromise that will allow for as great an approximation as possible is necessary. Using an increased number of measuring points in a randomized fashion is a method that has been used in current research, thus ensuring that extreme values do not dramatically alter the overall measurements and including more parts of the oftentimes inhomogeneous scar surface into the measurement [3]. There is, however, no consensus on how many measurements are necessary to adequately represent the results of a larger area. In their research, the authors have commonly used three measurements for an area of $10 \mathrm{~cm}$ by $10 \mathrm{~cm}$ when measuring skin elasticity or other parameters that rely on assessment through small probes (• Fig. 16.2).

\subsection{Interpreting Therapeutic Success with Objective Scar Assessment Technologies}

\subsubsection{Data Assessment and Evaluation}

After the measurements, the resultant data needs to be sorted and analyzed. To present the most objective results possible, it is necessary to use the raw abso- lute data for further analyses. Only including relative changes makes it hard for outside observers to interpret the importance of the findings when absolute baseline values are not known. Furthermore, the level of measurement that is required for certain statistical tests is not sufficient when using relative data.

Then, tests can be performed to elucidate the statistical significance of the findings.

\subsubsection{Clinically Important Difference}

After performing statistical testing, one must consider whether the results of the measurements constitute a clinically important difference. Currently, there are no clear recommendations on what constitutes a clinically important difference in objective scar assessment in the literature. However, ideally, every modern study on scar treatment and its effects can include its own control to evaluate whether the results are clinically important.

After all, scar treatment should alleviate the patients' symptoms, improve functional impairments, and address aesthetic disfigurements. Therefore, patientderived questionnaires like the POSAS Patient Scale or the Dermatology Life Quality Index (DLQI) can be included in the research as a control for patient satisfaction [4]. Should those questionnaires show statistically significant improvements, just like the objective assessment tools, then a clinically important difference has been achieved. While the lack of statistically significant results does not necessarily rule out a clinically important difference, it is important to consider just how sensitive modern assessment tools have become. Surface profilometry can measure changes at the micrometer scale, and other technologies boast similar sensitivities. But even if changes can be documented on such a fine scale, statistical significance of such results does not help the patients if they do not register improvements regarding their impairments.

Therefore, defining an absolute clinically important difference for objective scar assessment is neither possible nor sensible. The importance of documented improvements should always be compared to the patients' assessment of the therapeutic results to gauge the overall success.

\subsection{Conclusion}

Objective scar assessment has come a long way during the recent years. Today, a multitude of options for the documentation of scar treatment is available. From this variety of options, researchers and clinicians have to choose the tools best suited for their individual analysis based on the scar parameters that require documentation and the individual devices' features. Care should be taken to choose 
assessment technology that does not rely on proprietary assessment and evaluation formats but that is able to export results in standardized units of measurement so that comparability with other research groups and studies is ensured. Measurements should be performed in a standardized environment with well-maintained and well-calibrated tools, and conditions as well as patient positioning should not differ between measurements.

Objective scar assessment relies on using the most accurate values to represent the results which is why absolute values should always be preferred to relative values. Not only does this ensure comparability of the results, it allows for more complex statistical testing, thus ensuring improved significance of the findings.

A clinically important difference, however, cannot be derived from statistically significant findings alone. Here, the patient's perspective should be taken into consideration. As modern technology is now able to document extremely subtle changes, clinically important differences, despite significant statistical changes, can only be asserted if the patient considers them significant. This further illustrates that neither subjective nor objective scar assessment means alone should be employed. Both clinical and research protocols should include both to profit from additional information and to further characterize their findings.

\section{Take-Home Messages}

- Choosing the right objective scar assessment tools is paramount and should be based on the scar parameters that are central to the research.

- Devices that work with standardized units of measurement should be preferred to those that work with proprietary units or formats.
- Maintaining a standardized environment for the measurements both regarding the instruments (regular cleaning and calibration are required; the proper configuration should be checked before measurements are performed) and the patients (standardized posture, identical location for the measurements) is crucial.

- Absolute values should be preferred to relative values to improve the informative value and the statistical significance of the findings.

- The clinically important difference in objective scar assessment is rather a correlation between objective findings and subjective patient satisfaction, than a certain amount of improvement according to technical measurements. Modern assessment technology is oftentimes so sensitive that statistical testing reveals significant improvements before patients notice them.

\section{References}

1. Poetschke J, Schwaiger H, Gauglitz GG. Current and emerging options for documenting scars and evaluating therapeutic progress. Dermatol Surg. 2017;43 Suppl 1:S25-s36.

2. Roques C, Teot L, Frasson N, Meaume S. PRIMOS: an optical system that produces three-dimensional measurements of skin surfaces. J Wound Care. 2003;12(9):362-4.

3. Poetschke J, Dornseifer U, Clementoni MT, Reinholz M, Schwaiger H, Steckmeier S, et al. Ultrapulsed fractional ablative carbon dioxide laser treatment of hypertrophic burn scars: evaluation of an in-patient controlled, standardized treatment approach. Lasers Med Sci. 2017;32(5):1031-40.

4. Reinholz M, Poetschke J, Schwaiger H, Epple A, Ruzicka T, Gauglitz GG. The dermatology life quality index as a means to assess life quality in patients with different scar types. J Eur Acad Dermatol Venereol. 2015;29(11):2112-9.

Open Access This chapter is licensed under the terms of the Creative Commons Attribution 4.0 International License (http://creativecommons. $\mathrm{org} /$ licenses/by/4.0/), which permits use, sharing, adaptation, distribution and reproduction in any medium or format, as long as you give appropriate credit to the original author(s) and the source, provide a link to the Creative Commons license and indicate if changes were made.

The images or other third party material in this chapter are included in the chapter's Creative Commons license, unless indicated otherwise in a credit line to the material. If material is not included in the chapter's Creative Commons license and your intended use is not permitted by statutory regulation or exceeds the permitted use, you will need to obtain permission directly from the copyright holder. 